\title{
Association between micronutrients and maternal leukocyte telomere length in early pregnancy in Rwanda
}

Etienne Nsereko ${ }^{1 *}$, Aline Uwase $^{1}$, Claude Mambo Muvunyi ${ }^{2}$, Stephen Rulisa², David Ntirushwa², Patricia Moreland ${ }^{3}$, Elizabeth J. Corwin ${ }^{4}$, Nicole Santos ${ }^{5}$, Jue Lin ${ }^{6}$, Jyu-Lin Chen ${ }^{7}$, Manasse Nzayirambaho ${ }^{8}$ and Janet M. Wojcicki ${ }^{9,10^{*}}$

\begin{abstract}
Background: Exposure to environmental stressors can lead to shorter leukocyte telomere length and increase the risk of chronic diseases. Preservation of leukocyte telomere length by reducing oxidative stress exposure and reinforcing immunity may be a mechanism by which nutritional factors delay or prevent chronic disease development.

Methods: Healthy pregnant women (aged 18-45 years) at 9-15 weeks of gestation living in Gasabo District, Kigali, Rwanda, were recruited from 10 health centers for a prospective, longitudinal study from September to October 2017 to determine possible associations between nutrition health, infectious disease and leukocyte telomere length. Anthropometric and laboratory measurements were performed using standard procedures; sociodemographic parameters and health histories were assessed via surveys, and leukocyte telomere length was assessed using quantitative PCR expressed as the ratio of a telomeric product to a single-copy gene product (T/S).

Results: Mean gestational age of participants $(n=297)$ at enrollment was $13.04 \pm 3.50$ weeks, age was $28.16 \pm 6.10$ years and leukocyte telomere length was $1.16 \pm 0.22$ (T/S). Younger age; no schooling vs. primary schooling; and lower levels of ferritin, soluble transferrin receptors and retinol-binding protein were independent predictors of longer telomere length in multivariable models.

Conclusions: Leukocyte telomere length is an indicator of biological aging in pregnant Rwandan women. Maternal micronutrient status, specifically lower ferritin, soluble transferrin receptor levels, and retinol-binding protein levels were associated with longer maternal telomere length in contrast with some studies from North America and Europe. There were no associations between inflammation and infectious disease status and maternal leukocyte telomere length. Further studies are needed to enhance our understanding of the interplay between maternal nutritional status and infectious disease in relation to leukocyte telomere length in developing countries.
\end{abstract}

Keywords: Nutrition, Infection, Pregnancy, Oxidative stress, Leukocyte Telomere length

\footnotetext{
* Correspondence: etiennen70@gmail.com; wojcicki@gmail.com

${ }^{1}$ College of Medicine and Health Sciences School of Health Sciences, University of Rwanda, P.O. Box: 3538, Kigali, Rwanda

${ }^{9}$ Department of Pediatrics, University of California San Francisco, San Francisco, USA

Full list of author information is available at the end of the article
}

(c) The Author(s). 2020 Open Access This article is licensed under a Creative Commons Attribution 4.0 International License, which permits use, sharing, adaptation, distribution and reproduction in any medium or format, as long as you give appropriate credit to the original author(s) and the source, provide a link to the Creative Commons licence, and indicate if changes were made. The images or other third party material in this article are included in the article's Creative Commons licence, unless indicated otherwise in a credit line to the material. If material is not included in the article's Creative Commons licence and your intended use is not permitted by statutory regulation or exceeds the permitted use, you will need to obtain permission directly from the copyright holder. To view a copy of this licence, visit http://creativecommons.org/licenses/by/4.0/. The Creative Commons Public Domain Dedication waiver (http://creativecommons.org/publicdomain/zero/1.0/) applies to the data made available in this article, unless otherwise stated in a credit line to the data. 


\section{Background}

\section{Leukocyte telomeres and maternal health}

Telomeres are protective nucleoprotein structures that appear at the ends of chromosomes and confer protection against chromosomal damage [1]. They undergo attrition with each cell division due to limitations in the ability of the DNA replication machinery to replicate chromosome ends. Changes in telomeres serve as a biological marker for cellular aging, [2] with critical shortening resulting in cellular senescence [3]. Telomeres shorten throughout the normal aging process, and shorter telomere length has been associated with multiple chronic diseases, including cancer, type II diabetes, and cardiovascular disease [4]. Further, they are particularly sensitive to reactive oxygen species (ROS) damage; exposure to chronic or acute and social and environmental stressors can often lead to increased ROS exposure [3, 5].

Antioxidants, which maintain homeostasis via affecting redox status and/or redox-sensitive signaling pathways and gene expression, may help reduce ROS damage [5]. They play a specific role in boosting immune response and restricting pathological aspects of the cytokinemediated response [6, 7]. Shorter leukocyte telomere length is reportedly associated with micronutrient deficiencies and inadequate diet attributed to the insufficient intake of iron, vitamin B12 and D, and other vitamins and minerals $[8,9]$. During pregnancy, maternal nutritional health is pivotal as deficiencies of certain vitamins or micronutrients and poor dietary intake may affect fetal telomere length, consequently having an adverse effect on the health of the newborn child $[9,10]$.

\section{Nutrition and infection in pregnancy}

Malnutrition in pregnant, lactating women and in women of childbearing age is a common problem in sub-Saharan Africa (SSA) and is attributable to limited weight gain during pregnancy and micronutrient deficiencies [11, 12]. In Rwanda, anemia and low hemoglobin levels are present in $19 \%$ women of childbearing age, of which $7 \%$ are underweight $[13,14]$. In a recent study, during early pregnancy, anemia was found to be present in 33\% participants; in addition, participants showed deficiencies in iron levels as measured by ferritin and soluble transferrin receptors (sTfRs) (19.1\%) [15]. In the same study, 27.3\% and 7.9\% participants showed high levels of inflammatory markers; $C$ reactive protein (CRP) and alpha A glycoprotein (AGP) suggestive of infection or other inflammatory processes [15].

Systemic, sexually transmitted or genitourinary infections are common in SSA populations [16, 17]. In Rwanda, hepatitis B virus, co-infection with hepatitis B virus/human immunodeficiency virus (HIV), and syphilis affects $3.5 \%, 4.1 \%$, and $2 \%$ pregnant women, respectively [18]. Other infectious diseases such as malaria affect $5.7 \%$ of pregnant women [19]. Overall malaria burden is high with 403 cases per 1,000 in 2016 and annual incidence increasing since 2012 [20]. Maternal systemic inflammation during pregnancy has been associated with diverse adverse outcomes including fetal growth restriction, and preterm birth [1, 21, 22], but little is known about its association with maternal leukocyte telomere length, particularly in SSA, where malnutrition and infection during pregnancy are more common than North American or European contexts.

To the best of our knowledge, no studies have investigated the relationship between maternal nutritional status, infection, and maternal leukocyte telomere length in SSA. In this study, we evaluated the association between nutritional and infectious disease risk factors and leukocyte telomere length during early pregnancy in women from Gasabo District, Rwanda.

\section{Methods}

\section{Participants and inclusion criteria}

We assessed leukocyte telomere length in healthy pregnant women aged 18-45 years as part of a prospective, cohort study of pregnant women from 10 health centers in Gasabo District, Kigali Province [15]. Participants were enrolled from September to October 2017 as previously described [15] and were in early pregnancy (gestational age $9-15$ weeks, as confirmed by ultrasound and self-reported last menstrual period). Inclusion criteria included testing negative for HIV and syphilis, singleton pregnancy, speaking Kinrywanda or English and providing written consent for participation. Out of 420 participants, 300 were included in the telomere substudy based on convenience sampling. Two women were excluded due to unclear labeling of the samples and 1 did not complete the study due to loss to follow-up. Leukocyte length was assessed in 297 participants. The participating health centers were categorised into rural or urban and each contributed $50 \%$ of the participants.

\section{Recruitment procedure and ethical approval}

Pregnant women were approached by community health workers in charge of maternal and child healthcare in the catchment area under their responsibilities as previously described in detail [15]. Eligible candidates reported to the nearest health center for preliminary screening, and trained enumerators obtained verbal and written informed consent from participants prior to their inclusion in the study.

The Institutional Review Board (IRB) of the University of Rwanda and Committee on Human Research (CHR) at the University of California, San Francisco, approved the study protocol.

\section{Data collection}

After participant enrollment, trained phlebotomists collected whole blood samples, and six trained interviewers 
conducted verbal interviews to collect data on sociodemographic parameters and performed anthropometric measurements. Four on-site laboratory technicians separated blood for micronutrient analyses and processed laboratory specimens.

\section{Infection and nutritional assessment}

Serum and whole blood samples were frozen at $-80^{\circ} \mathrm{C}$ at the University Teaching Hospital of Kigali before shipping them to VitMin Lab (NutriSurvey) in Germany for analyses of micronutrients and inflammatory markers. Using the combined sandwich enzyme-linked immunosorbent assay technique [23], samples were analyzed for sTfRs, RBP, ferritin, AGP, and CRP. For data interpretation, we used cutoff point values recommended by the World Health Organization (WHO): anemia (hemoglobin $<11 \mathrm{~g} / \mathrm{dL}$ ) [24], low serum ferritin < $12 \mu \mathrm{g} / \mathrm{L}$, and $\mathrm{RBP}<0.83 \mu \mathrm{mol} / \mathrm{L}$. Further, serum concentrations of $>5 \mathrm{mg} / \mathrm{L}$ CRP and $>1$ AGP served as acute and chronic markers of inflammation, respectively [25].

\section{Leukocyte telomere length analysis}

Whole blood samples were shipped to the Elizabeth Blackburn Laboratory at the University of California, San Francisco, for leukocyte telomere length analysis. DNA was extracted using the QIAamp DNA Investigator Kit (cat. no. 56,504; QIAGEN), and leukocyte telomere length was determined using quantitative PCR. The assay to measure telomere length was an adaptation of the original method published by Cawthon [26], as presented by Lin et al. [27]. Telomere length is expressed as $\mathrm{T} / \mathrm{S}$, representing the ratio of a telomeric product to a single-copy gene product [27]. The average coefficient of variation for these samples were $2.1 \%$.

\section{Screening for genitourinary infections}

Vaginal swabs were collected from all participants and examined for genitourinary infections. Cultures were grown and biochemical identification was performed, according to standard procedures [28]. As previously described [15], Trichomonas vaginalis presence was determined using wet mount microscopy [29], while Candida albicans presence was determined by cultures, followed by the germ tube test [30]. Chlamydia trachomatis was detected using CORTEZ One-Step Chlamydia Rapicard $^{\mathrm{m}}$ [31]. The culture method used for detecting Neisseria gonorrhea was not successful.

Urinary tract infection was diagnosed by growing cultures using standard procedures [32], and the final identification process involved using the catalase test and analytical profile index (API 20E) [33].

\section{Demographic, dietary, and anthropometric data}

Questionnaires were administered to collect demographic data, including maternal age, education level, residence, socioeconomic status, partnership/marital status, and reproductive history. The questionnaire covered food frequency items necessary to evaluate the Minimum Dietary Diversity for Women [34, 35].

A standardized digital scale and portable stadiometer were used to measure the weight and height, respectively, of participants, and body mass index (BMI) was then calculated. Following the WHO and Centers for Disease Control guidelines [36], BMI was used to categorize women as follows: $<18.5 \mathrm{~kg} / \mathrm{m}^{2}=$ underweight; 18.5 to $<25 \mathrm{~kg} / \mathrm{m}^{2}=$ normal weight; 25.0 to $<30 \mathrm{~kg} / \mathrm{m}^{2}=$ overweight; and $\geq 30.0 \mathrm{~kg} / \mathrm{m}^{2}=$ obese. The mid-upper arm circumference (MUAC) was recorded, which served as an indirect indicator of nutritional status. MUAC $<$ $23 \mathrm{~cm}$ indicated nutritional deficiency, while MUAC > = $23 \mathrm{~cm}$ indicated nutritional sufficiency [37].

\section{Statistical analysis}

Data were checked for consistency using Excel 16.5 and then coded and exported to SPSS v20 for analyses. Normality of continuous variables was graphically assessed using Quantile-Quantile (QQ), Probability-Probability (PP) plots and histograms. Test indicated that leukocyte telomere length has a normal distribution (Fig. 1). Simple and multiple linear regressions were used to assess possible relationships for all predictors, including maternal nutrition and infectious disease status with maternal leukocyte telomere length. Means and standard deviations were calculated for predictors of interest. All variables with significance at $\mathrm{p} \leq 0.15$ were included in multivariable models. Backward stepwise regression was used, with significance set at $p<0.05$; similarly, multivariable models had significance set at $p<0.05$. Variables that were collinear $(r>0.7)$ were not included in the models concurrently, including presence of Chlamydia trachomatis bacterial infection and other sexually transmitted infections. Results are reported as slope $(\beta)$ with 95\% confidence interval (CI) estimated by final multivariable models using linear multivariable regression and backward stepwise technique.

\section{Results}

Sociodemographic, anthropometric and behavioral data and leukocyte telomere length

Mean gestational age at enrollment was $13.04 \pm 3.50$ weeks for 297 participants. Mean maternal age was $28.16 \pm 6.10$ years, and mean leukocyte telomere length was $1.16 \pm 0.22 \mathrm{~T} / \mathrm{S}$. Most participants $(82.2 \% ; n=244)$ were between 20 and 35 years, and for this age category, the mean leukocyte telomere length was $1.17 \pm 0.22 \mathrm{~T} / \mathrm{S}$ (Table 1). Maternal age had a significantly negative 


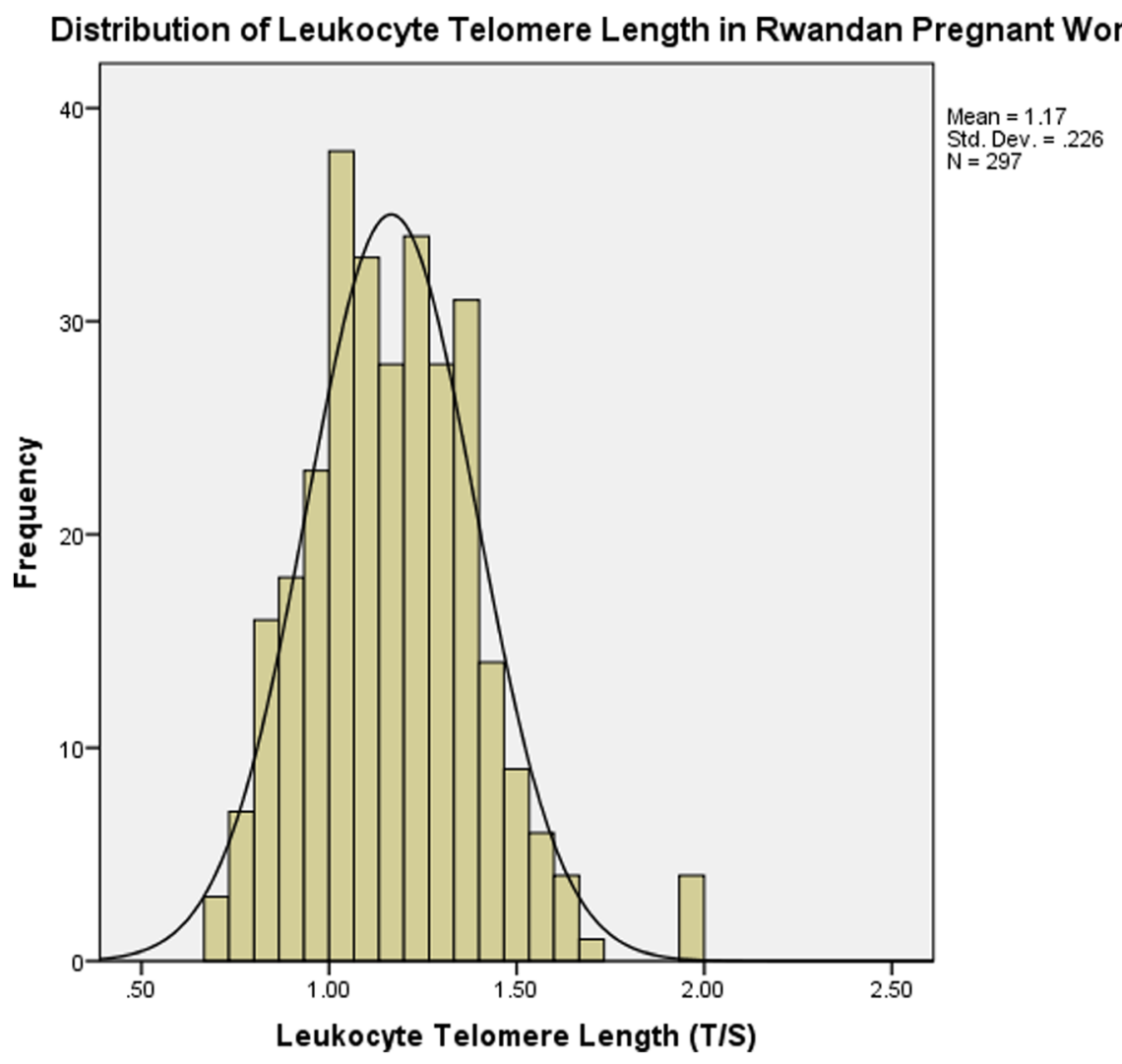

Fig. 1 Distribution of Leukocyte Telomere Lenght among pregnant women in Rwanda

association with maternal leukocyte telomere length ( $\beta=$ $-2.96 ; p=0.003)$. No association was found between residential area within Gasabo District (urban vs. rural), occupation (employed vs. not working), and marital status (living with a partner vs. living alone) and maternal leukocyte telomere length (Table 1). Participants who had secondary schooling or higher had longer telomeres than those who did not ( $\beta=0.17$, 95\% CI: $0.03-0.14 ; p=0.003$; Table 1). Primary schooling was associated with shorter leukocyte telomere length as compared with no education (Table 1).

BMI of most participants was within the normal range (73.4\%; $n=215)$, with the mean value $23.09 \pm 3.39 \mathrm{~kg} / \mathrm{m}^{2}$. An increase in BMI measured continuously was associated with a decrease in overall leukocyte telomere length $(\beta=-2.11$, $95 \%$ CI: -0.02 to $0.01 ; p=0.03)$. No association was found between alcohol consumption and maternal leukocyte telomere length, although self-reported gender-based violence trended towards significance with shorter telomere length ( $\beta=0.10,95 \%$ CI: -0.24 to $012 ; p=0.08$ ).

\section{Obstetrical history, pregnancy outcomes and telomere length}

The majority of the cohort was multiparous $(n=178$, 59.9\%) and had not experienced stillbirth $(n=289$,
96.3\%). Most participants had not undergone a cesarean section ( $n=261,90.9 \%$ ), experienced previous preterm labor $(n=246,82.8 \%)$, or suffered a miscarriage $(n=250$, $85.6 \%)$. Most participants had a vaginal delivery $(n=239$, $80.5 \%$ ) with the current pregnancy.

In total, 9.1\% $(n=27)$ of participants experienced preterm birth (gestational age $<37$ weeks). The mean gestational age of the cohort was $38.8 \pm 2.1$ weeks, and for participants with preterm neonates, it was $33.6 \pm 2.6$ weeks. Participants who delivered before 34 weeks of gestation represented $3.7 \%$ of the cohort $(n=11)$, while those who delivered from 34 to 36 weeks of gestation represented $5.4 \%(n=16)$ of the cohort. No statistically significant association was found between maternal obstetrical history or delivery-related variables, including previous preterm birth and gestational age, and maternal leukocyte telomere length (Table 2).

\section{Inflammation and infections in pregnancy and leukocyte telomere length}

Overall, 26\% of the cohort $(n=77)$ had acute inflammation as suggested by elevated CRP levels and $7.4 \%$ had chronic inflammation as indicated by elevated AGP level (Table 3). There was no association between maternal leukocyte telomere length and CRP levels, AGP levels, 
Table 1 Social demographic/behavioral characteristics and leukocyte telomere length $(N=297)$

\begin{tabular}{|c|c|c|c|c|c|}
\hline & \multicolumn{2}{|l|}{ Descriptive } & \multicolumn{3}{|c|}{ Simple linear regression } \\
\hline & Total N (\%) or mean +/-SD & Leukoctye telomere length mean+/-SD(T/S) & $\beta$ & $\mathrm{Cl}$ & $p$ \\
\hline Age in years & $28.16 \pm 6.10$ & $1.16 \pm 0.22$ & -2.96 & {$[.010 ;-0.02]$} & $0.003^{* *}$ \\
\hline \multicolumn{6}{|l|}{ Age } \\
\hline$<20$ & $13(4.3)$ & $1.18 \pm 0.21$ & Ref. & & \\
\hline$\geq 20-35$ & $244(82.2)$ & $1.17 \pm 0.22$ & -0.11 & {$[-021 ; 0.06]$} & 0.31 \\
\hline$>35$ & $40(13.5)$ & $1.11 \pm 0.22$ & -0.14 & {$[-013 ; 0.11]$} & 0.90 \\
\hline Gestational age (weeks) at recruitment & $13.04 \pm 3.5$ & & 0.012 & {$[0.01 ; 0.2]$} & 0.74 \\
\hline \multicolumn{6}{|l|}{ Residence } \\
\hline Urban & $104(35.0)$ & $1.17 \pm 0.21$ & Ref. & & \\
\hline Rural & $193(65.0)$ & $1.14 \pm 0.25$ & 0.5 & {$[-03 ; 0.08]$} & 0.32 \\
\hline \multicolumn{6}{|l|}{ Occupation } \\
\hline Paying Job & $150(50.5)$ & $1.19 \pm 0.23$ & refer & & \\
\hline Unemployed & $147(49.5)$ & $1.17 \pm 0.22$ & 0.8 & {$[-0.01 ; 0.05]$} & 0.14 \\
\hline \multicolumn{6}{|l|}{ Living with a partner $\left(n^{\mathrm{a}}=296\right)$} \\
\hline Yes & $273(92.2)$ & $1.17 \pm 023$ & Ref. & & \\
\hline No & $23(7.8)$ & $1.11 \pm 0.16$ & -0.06 & {$[-0.15 ; 0.04]$} & 0.26 \\
\hline \multicolumn{6}{|l|}{ Educational level } \\
\hline Never schooled & $146(49.2)$ & $1.15 \pm 0.23$ & Ref. & & \\
\hline Primary & $61(20.5)$ & $1.06 \pm 0.18$ & -0.16 & {$[-0.16 ;-0.3]$} & $.005^{* *}$ \\
\hline Secondary or higher & $90(30.3)$ & $1.24 \pm 0.21$ & 0.17 & {$[0.03 ; 0.14]$} & $.003^{*}$ \\
\hline Body Mass Index (BMI) (kg/m²) & 23.09 (3.39) & $1.16 \pm 0.22$ & -2.11 & {$[-0.02 ; 0.01]$} & $0.03^{*}$ \\
\hline \multicolumn{6}{|l|}{ BMI (category) $\left(n^{\mathrm{a}}=293\right)$} \\
\hline Underweight & $11(3.8)$ & $1.12 \pm 0.26$ & Ref. & & \\
\hline Normal Weight & $215(73.4)$ & $1.18 \pm 0.22$ & 0.10 & {$[-0.06 ; 0.17]$} & 0.39 \\
\hline Overweight & $55(18.7)$ & $1.11 \pm 0.23$ & -0.04 & {$[-0.15 ; 0.11]$} & 0.73 \\
\hline Obese & $12(4.1)$ & $1.16 \pm 0.25$ & 0.31 & {$[-014 ; 0.19]$} & 0.75 \\
\hline \multicolumn{6}{|l|}{ Alcohol use (at least 2-4 times per month) } \\
\hline No & $228(76.8)$ & $1.17 \pm 0.25$ & Ref. & & \\
\hline Yes & $69(23.2)$ & $1.16 \pm 0.21$ & 0.01 & {$[-0.05 ; 0.06]$} & 0.83 \\
\hline \multicolumn{6}{|l|}{ Gender-based Violence } \\
\hline Yes & $12(4.0)$ & $1.17 \pm 0.22$ & Ref. & & \\
\hline No & $285(96.0)$ & $1.05 \pm 016$ & 0.10 & {$[-0.24 ; 0.01]$} & 0.08 \\
\hline
\end{tabular}

${ }^{*} p<0.01,{ }^{*} p<0.05$

$\mathrm{n}^{\mathrm{a}}$ Sample size reduced by missing responses among covariates

or presence of urinary tract infections (Table 3). Having a sexually transmitted infection during pregnancy and shorter maternal telomere length approached statistical significance $(\beta=-0.104,95 \% \mathrm{CI}:-0.102$ to $0.005 ; p=$ 0.07 ), as did having a bacterial Chlamydia trachomatis infection ( $\beta=-0.010,95 \% \mathrm{CI}:-0.12$ to $0.01 ; p=0.08$; Table 3).

\section{Nutrition in pregnancy and leukocyte telomere length}

A significant number $(n=92,31 \%)$ of participants had anemia during pregnancy; $3.4 \%(n=10)$ with a lower percentage having iron deficiency as indicated by ferritin or soluble transferrin receptors deficiency (Table 4). Nineteen-point two percent of participants had ferritin deficiency $(n=58)$ with $3.4 \%(n=10)$ having transferrin receptor deficiency (Table 4). Continuous measures of sTfR levels were inversely associated with telomere length ( $\beta=-0.13,95 \% \mathrm{CI}:-0.03$ to $0.01 ; p=0.02)$, as were RBP levels ( $\beta=-2.19,95 \%$ CI: -0.13 to $0.01 ; p=$ 0.03). Deficiencies in sTfR levels $(>8.3 \mathrm{mg} / \mathrm{L})$ approached statistical significance, with a negative association between deficiency and longer leukocyte telomere length ( $\beta=-0.10,95 \% \mathrm{CI}:-0.26$ to $0.17 ; p=0.08$; Table 4). No association was present for other markers 
Table 2 Obstetrical history, pregnancy outcomes and leukocyte telomere length $(n=297)$

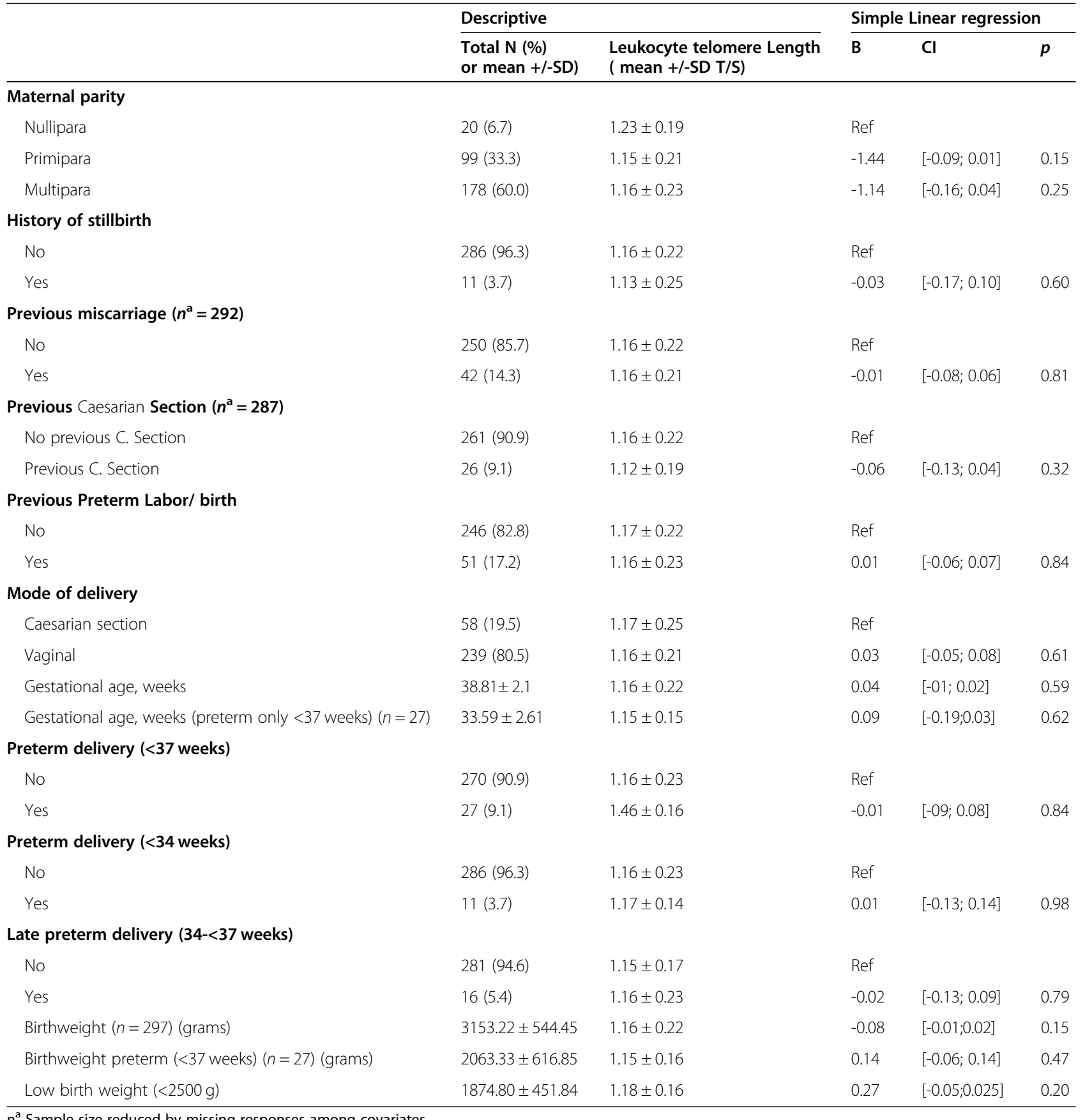

of maternal nutritional health, including RBP deficiency, ferritin deficiency, inadequate dietary diversity, indicators of malnutrition and maternal leukocyte telomere length (Table 4).

\section{Multivariable linear regression}

Independent predictors of longer leukocyte telomere length included younger maternal age $(\beta=-0.14,95 \% \mathrm{CI}$ : -0.01 to $-0.001 ; p=0.01$ ), no schooling vs. primary schooling ( $\beta=-0.19,95 \% \mathrm{CI}:-0.17$ to $-0.041 ; p=0.002)$, lower levels of sTfRs $(\beta=-0.15,95 \%$ CI: -0.04 to -0.007 ; $p<0.01)$, and lower levels of ferritin $(\beta=-0.13,95 \% \mathrm{CI}$ : -0.01 to $-0.001 ; p=0.02$; Table 5$)$. Similarly, in the backward stepwise regression model, independent predictors of longer telomere length were younger age $(\beta=-0.15$, 95\% CI: -0.01 to $-0.001 ; p<0.01)$, absence of primary schooling ( $\beta=-0.21 ; 95 \% \mathrm{CI}:-0.18$ to $-0.05 ; p<0.01$ ), lower levels of sTfRs ( $\beta=-0.15$, 95\% CI: -0.04 to -0.007 ; 
Table 3 Maternal inflammation, infection and leukocyte telomere length ( $n=297)$

\begin{tabular}{|c|c|c|c|c|c|}
\hline & \multicolumn{2}{|l|}{ Descriptive } & \multicolumn{3}{|c|}{ Simple Linear regression } \\
\hline & Total $\mathrm{N}(\%)$ or mean \pm SD) & Leukocyte telomere Length mean \pm SD (T/S) & B & $\mathrm{Cl}$ & $P$ \\
\hline \multicolumn{6}{|c|}{ Markers of Acute and Chronic Inflammation } \\
\hline C Reactive Protein (CRP mg/l) & $5.08 \pm 12.33$ & $1.16 \pm 0.22$ & 0.02 & {$[-0.01 ; 0.03]$} & 0.67 \\
\hline \multicolumn{6}{|l|}{$\operatorname{CRP}(>5 \mathrm{mg} / \mathrm{l})\left(n^{\mathrm{a}}=296\right)$} \\
\hline No & $219(74.0)$ & $1.16 \pm 0.23$ & ref & & \\
\hline Yes & $77(26)$ & $1.17 \pm 0.21$ & 0.04 & {$[-0.04 ; 0.07]$} & 0.53 \\
\hline$a_{1}$-acid glycoprotein (AGPg/dl) & $0.61 \pm 0.41$ & $1.16 \pm 0.22$ & -0.45 & {$[-0.07 ; 0.04]$} & 0.64 \\
\hline \multicolumn{6}{|l|}{$a_{1}$-acid glycoprotein (AGP>1 g/l) } \\
\hline No & $275(92.6)$ & 1.17. \pm 0.23 & ref & & \\
\hline Yes & $22(7.4)$ & $1.13 \pm 0.15$ & -0.04 & {$[-0.13 ; 0.06]$} & 0.48 \\
\hline \multicolumn{6}{|l|}{ Presence of Infectious Disease } \\
\hline \multicolumn{6}{|l|}{ Urinary Tract Infections (UTI) } \\
\hline No & $259(87.2)$ & $1.17 \pm 0.23$ & Ref & & \\
\hline Yes & $38(12.8)$ & $1.16 \pm 0.16$ & -0.01 & {$[-0.08 ; 0.07]$} & 0.84 \\
\hline \multicolumn{6}{|l|}{ UTI strain $(n=38)$} \\
\hline Stenotrophomonas maltophilia & $2(5.3)$ & & & & \\
\hline Escherchia coli & $22(57.9)$ & & & & \\
\hline Klebsialla pneumoniae & $11(28.9)$ & & & & \\
\hline Staphylococcus epidermitis & $3(7.9)$ & & & & \\
\hline \multicolumn{6}{|c|}{ Sexually Transmitted Infection (STI) } \\
\hline No & $189(63.6)$ & $1.18 \pm 0.23$ & ref & & \\
\hline Yes & $108(36.4)$ & $1.13 \pm 0.22$ & -0.104 & {$[-0.102 ; 0.005]$} & 0.07 \\
\hline \multicolumn{6}{|l|}{ Type of STI } \\
\hline \multicolumn{6}{|l|}{ Chlamydia trachomatis } \\
\hline No & $235(79.1)$ & $1.17 \pm 0.22$ & ref & & \\
\hline Yes & $62(20.9)$ & $1.12 \pm 0.25$ & -010 & {$[-0.12 ; 0.01]$} & 0.08 \\
\hline \multicolumn{6}{|l|}{ Trichomonas vaginalis } \\
\hline No & $282(94.9)$ & $1.16 \pm 023$ & ref & & \\
\hline Yes & $15(5.1)$ & $1.17 \pm 0.19$ & 0.002 & {$[-0.11 ; 0.12]$} & 0.99 \\
\hline \multicolumn{6}{|l|}{ Candida albicans } \\
\hline No & $233(78.5)$ & $1.17 \pm 0.23$ & ref & & \\
\hline Yes & $64(21.5)$ & $1.14 \pm 0.19$ & -0.51 & [-0.09; 0.03] & 0.25 \\
\hline
\end{tabular}

$\mathrm{n}^{\mathrm{a}}$ Sample size reduced by missing responses among covariates

$p<0.01)$, lower levels of ferritin $(\beta=-0.12,95 \%$ CI: -0.01 to $-0.001 ; p=0.027)$, and lower levels of RBP $(\beta=-0.11$, 95\% CI: -0.12 to $-0.001 ; p=0.04$; Table 5).

\section{Discussion}

This is the first study to assess association between maternal infections, nutritional health and leukocyte telomere length in pregnant women in SSA. Furthermore, it is the first study of leukocyte telomere length among women in Rwanda. We found evidence of leukocyte telomere length as a marker of biological aging in Rwandan women and also of possible associations between maternal micronutrient status and leukocyte telomere length in pregnant women in Rwanda. Other leukocyte telomere length studies with pregnant women have been conducted primarily in the European and North American context, which presents a different environmental milieu in terms of overall burden of infectious disease and maternal nutritional health $[38,39]$. In contrast with these other studies, the endemicity of infectious diseases in Kigali, Rwanda, including clinical and subclinical infections such as a higher prevalence of sexually transmitted infections and endemicity of malaria, provides a different context to assess the association between maternal leukocyte telomere length and birth outcomes [40]. 
Table 4 Maternal nutrition and leukocyte telomere length $(n=297)$

\begin{tabular}{|c|c|c|c|c|c|}
\hline & \multicolumn{2}{|l|}{ Descriptive } & \multicolumn{3}{|c|}{ Simple Linear regression } \\
\hline & $\begin{array}{l}\text { Total } N(\%) \text { or } \\
\text { mean } \pm \text { SD) }\end{array}$ & $\begin{array}{l}\text { Leukocyte telomere length } \\
\text { mean } \pm S D(T / S)\end{array}$ & $\beta$ & $\mathrm{Cl}$ & $P$ \\
\hline Hemoglobin (g/dl) & $11.23 \pm 1.13$ & $1.16 \pm 0.22$ & 0.04 & {$[-0.01 ; 0.03]$} & 0.47 \\
\hline \multicolumn{6}{|l|}{ Anemia (<11 g/dl) } \\
\hline No & $205(69.0)$ & $1.15 \pm 0.21$ & Ref & & \\
\hline Yes & $92(31.0)$ & $1.19 \pm 0.24$ & 0.07 & {$[-02 ; 0.09]$} & 0.21 \\
\hline sTFR (mg/L) (Soluble Transferrin Receptors) & $4.81 \pm 1.49$ & $1.16 \pm 0.22$ & -0.13 & {$[-0.03 ;-0.01]$} & $0.02^{*}$ \\
\hline \multicolumn{6}{|l|}{$s^{T F R}$ (mg/L) Deficiency } \\
\hline No deficiency ( $\leq \mathbf{8 . 3}$ mg/L) & 287 (96.6) & $1.17 \pm 0.22$ & Ref & & \\
\hline Deficiency (>8.3 mg/L) & $10(3.4)$ & $1.04 \pm 0.18$ & -0.10 & {$[-0.26 ; 0.17]$} & 0.08 \\
\hline Retinol Binding Protein (RBP) ( $\mu \mathrm{mol} / \mathrm{L})$ & $1.38 \pm 0.40$ & $1.16 \pm 0.22$ & -2.19 & {$[-0.13 ;-0.01]$} & $0.03^{*}$ \\
\hline \multicolumn{6}{|l|}{$\mathrm{RBP}(\mu \mathrm{mol} / \mathrm{L})$ Deficiency } \\
\hline No deficiency $(\geq 0.83 \mu \mathrm{mol} / \mathrm{L})$ & $239(80.5)$ & $1.17 \pm 0.18$ & Ref & & \\
\hline Deficiency $(<0.83 \mu \mathrm{mol} / \mathrm{L})$ & $58(19.5)$ & $1.16 \pm 0.23$ & 0.03 & {$[-0.05 ; 0.08]$} & 0.66 \\
\hline Ferritin $(\mu \mathrm{g} / \mathrm{L})$ & 73.52 & $1.16 \pm 0.22$ & -0.09 & {$[-0.01 ; 0.03]$} & 0.12 \\
\hline \multicolumn{6}{|l|}{ Ferritin Deficiency $(<12 \mu \mathrm{g} / \mathrm{L})$} \\
\hline No Deficiency $(<12 \mu \mathrm{g} / \mathrm{L})$ & $240(80.8)$ & $1.16 \pm 0.22$ & Ref & & \\
\hline Deficiency $(\geq 12 \mu \mathrm{g} / \mathrm{L})$ & $58(19.2)$ & $1.17 \pm 0.23$ & -0.01 & {$[-0.07 ; 0.06]$} & 0.84 \\
\hline Minimum Dietary Diversity for Women (MDDW) & $4.61 \pm 1.68$ & $1.16 \pm 0.22$ & -0.03 & {$[-0.06 ; 0.3]$} & 0.56 \\
\hline \multicolumn{6}{|l|}{ MDDW Deficiency } \\
\hline Adequate MDDW & $145(48.8)$ & $1.15 \pm 0.22$ & Ref & & \\
\hline Low MDDW & $152(51.2)$ & $1.17 \pm 0.23$ & -0.03 & {$[-0.07 ; 0.04]$} & 0.50 \\
\hline Mid-Upper Arm Circumference (MUAC) d(cm)(Mean +/-SD) & $25.72 \pm 3.06$ & $1.16 \pm 0.23$ & -0.09 & {$[-0.02 ; 0.002]$} & 0.30 \\
\hline \multicolumn{6}{|l|}{ MUAC Indication of Malnutrition } \\
\hline$\geq 23 \mathrm{~cm}$ & $236(79.5)$ & $1.16 \pm 0.23$ & Ref & & \\
\hline$<23 \mathrm{~cm}$ & $58(19.5)$ & $1.18 \pm 0.22$ & -0.04 & {$[-0.09 ; 0.04]$} & 0.45 \\
\hline
\end{tabular}

\section{Maternal age, leukocyte telomere length and Rwandan} women

A strong inverse association was found between maternal age and leukocyte telomere length, which is consistent with results of previous studies [4, 41, 42] demonstrating that leukocyte telomere length is a biological marker for aging in Rwandan women [41, 43]. This is the first study to our knowledge conducted of leukocyte telomere length in Rwandan women. The age range in our study was relatively narrow with the majority between 20 and 35 years of age (mean $28.16 \pm 6.10$ ) and even within this range, maternal age was highly predictive of leukocyte telomere length adjusting for other factors. Previous studies have found that Africans have longer leukocyte telomere length than Caucasians [44]; our results for reproductive age women are similar to those reported from a large population-based survey in United States for a similar age range of women and comparable to the African-Americans surveyed [45].

\section{Maternal nutritional status}

Several micronutrient markers were independently associated with shorter maternal leukocyte telomere length, after adjusting for other predictors. Specifically, we found associations between body iron stores as measured by lower numbers of soluble transferrin receptors, ferritin levels, and longer leukocyte telomere length. However, we found no associations between anemia and iron deficiency and leukocyte telomere length. Previous findings have been mixed including a study involving American adults ( $>65$ years) in whom high ferritin levels were associated with shorter telomere length [46] and a large study with British adults where transferrin saturation was associated with shorter leukocyte telomere length [47]. In contrast, another study found that iron consumption, as measured by dietary intake, was associated with longer telomere length in adults [48]. Importantly, the context where our study was conducted has different environmental stressors than in the previously 
Table 5 Multiple linear regression for determinants of maternal leukocyte telomere length $(n=297)$

\begin{tabular}{|c|c|c|c|c|c|c|c|c|}
\hline & \multicolumn{4}{|c|}{ Linear regression model full model } & \multicolumn{4}{|c|}{ Adjusted model } \\
\hline & B & SE & $\mathrm{Cl}$ & $P$ & $\beta$ & SE & $\mathrm{Cl}$ & $P$ \\
\hline Age (years) & -0.140 & 0.02 & {$[-0.01 ;-0.001]$} & 0.01 & -0.15 & 0.02 & {$[-0.01 ;-0.001]$} & $0.008^{* *}$ \\
\hline \multicolumn{9}{|l|}{ Occupation } \\
\hline Paying Job & & & & ref & & & & \\
\hline Housewife & -0.002 & 0.025 & {$[-0.05 ; 0.048]$} & 0.97 & - & - & - & - \\
\hline \multicolumn{9}{|l|}{ Education Level } \\
\hline Never schooled & & & & ref & & & & \\
\hline Primary & -0.190 & 0.033 & {$[-0.17 ; 0.041]$} & 0.002 & -0.21 & 0.033 & {$[-0.18 ;-0.05]$} & $0.001^{* *}$ \\
\hline Secondary or higher & 0.122 & 0.030 & {$[-0.001 ; 0.12]$} & 0.045 & 0.11 & 0.109 & {$[-0.004 ; 0.11]$} & .070 \\
\hline Body Mass Index (BMI) kg/m² & -0.094 & 0.004 & {$[-0.014 ; 0.01]$} & 0.101 & - & - & - & - \\
\hline \multicolumn{9}{|l|}{ Gender Based Violence (GBV) } \\
\hline Yes & & & & ref & & & & \\
\hline No & -0.107 & 0.063 & {$[-0.25 ; 0.01]$} & 0.053 & - & - & - & - \\
\hline Parity Nullipara(reference) & & & & ref & - & - & - & - \\
\hline Primipara & -0.020 & 0.055 & {$[-0.125 ; 0.09]$} & 0.75 & - & - & - & - \\
\hline Multipara & 0.046 & 0.027 & {$[.0 .03 ; 0.074]$} & 0.430 & - & - & - & - \\
\hline Birth weight & 0.053 & .0001 & {$[-0.01 ; 0.001]$} & 0.350 & & & & \\
\hline \multicolumn{9}{|l|}{ Sexually transmitted Infections } \\
\hline No & & & & ref & & & & \\
\hline Yes & -0.071 & 0.026 & {$[-0.84 ; 0.018]$} & 0.202 & & & & \\
\hline sTFR (mg/L) & -0.156 & 0.009 & {$[-0.04 ; 0.007]$} & 0.006 & -015 & 0.008 & {$[-0.04 ;-0.007]$} & $0.005^{* *}$ \\
\hline Retinol Binding Protein (RBP) ( $\mu \mathrm{mol} / \mathrm{L})$ & -0.093 & 0.031 & {$[-0.11 ; 0.010]$} & 0.098 & -0.11 & 0.03 & {$[-0.12 ;-0.001]$} & 0.04 \\
\hline Ferritin $(\mu \mathrm{g} / \mathrm{L})$ & -0.130 & .0001 & {$[-0.01 ; 0.001]$} & 0.023 & -0.124 & 0.001 & {$[-0.01 ;-0.001]$} & $0.027^{*}$ \\
\hline
\end{tabular}

${ }^{* *} p<0.01,{ }^{*} p<0.05$

described studies. As malaria infection is common in East Africa, including Rwanda, lower iron levels have been found to be associated with a reduced susceptibility to malaria infection in pregnant women [49]. Lower iron levels may have indirect health benefits in the context of areas with endemic malaria or other high incidence areas.

Alternatively, increased concentrations of iron can cause oxidative stress, consequently leading to telomere shortening [50]. Iron is a redox-active transitional metal with pro-oxidant properties [51]. Excessive iron during pregnancy can lead to ROS overproduction and oxidative stress [46]. Administering iron supplements to pregnant women is common as part of antenatal care services [14]; some pregnant Rwandan women may receive iron supplements to tackle iron deficiency, eventually leading to excessive iron in the body.

We also found an inverse relationship between RBP, an indirect indicator of retinol (vitamin A) levels, and leukocyte telomere length. As vitamin A is one of the antioxidants that provides a buffer against oxidative stress, it may protect against accelerated leukocyte telomere shortening [52]. Our results were unexpected and differ from those of other studies in which retinol levels were found to be positively associated with leukocyte telomere length [48]; moreover, vitamin A supplementation has been reported to be associated with longer telomere length [53]. However, these previous studies were not conducted with pregnant women in the first trimester of pregnancy. High levels of vitamin A, particularly during the first trimester, are associated with congenital malformations of the central nervous and cardiovascular systems [54], and high levels of serum retinol during pregnancy are associated with postpartum depression [55]. Elevated serum retinol levels may thus serve as an adverse risk marker of maternal and neonatal outcomes; further studies with multiple timepoints in pregnancy are warranted to assess direct effects.

Neither maternal BMI, MUAC or dietary intake measured by the MDDW index were associated with leukocyte telomere length. Our findings differ from the results of other studies that have consistently found maternal BMI levels associated with leukocyte telomere length $[56,57]$ as well as those that suggest that improved nutrition is associated with longer leukocyte telomere length [47]. In this study, a low percentage of women were obese (4.1\%) 
and underweight (3.8\%), with less heterogeneity in BMI compared with other population groups potentially explaining the contradictory results. Excess adipose tissue in obesity has been associated with shorter leucocyte telomere length but in North American contexts where obesity is more prevalent [58]. Additionally, our measurements were taken during early pregnancy which could have biased findings in contrast with other studies that have use pre-pregnancy BMI. Few studies measured weight during pregnancy in resource-poor settings in relation to maternal leucocyte telomere length.

\section{Infections and inflammation}

We found no association between biomarkers for inflammation and infection including sexually transmitted infections and leukocyte telomere length, although in bivariate analyses, any presence of sexually transmitted infection and infection with chlamydia trachomatis approached statistical significance for shorter leukocyte telomere length. Previous studies of infectious disease, specifically HIV in South Africa, have found shorter leukocyte telomere length with infection [59] although other studies with HIV infected individuals did not find any association, primary in the context of antiretroviral therapy immune reconstitution [38, 60]. Additional studies evaluating multiple pathogens have found that the association between shorter leukocyte telomere and infection may be pathogen specific [61]. We also assessed the association between general markers of inflammation and leukocyte telomere length as these have previously been found associated with shorter telomere length [39]. However, we did not assess duration or timing of infection and as such we may have not had sufficient information to assess associations with leukocyte telomere length. CRP levels rise sharply during the early phase of an infection, whereas AGP levels gradually increase so timing of testing may be critical to assess levels [62]. Furthermore, other studies were not conducted with pregnant women and CRP levels increase and AGP decrease with duration of gestation [21]. As such, the interplay between inflammatory status and leukocyte telomere length may not be fully understood in the context of pregnancy. Alternatively, it is possible that the cutoff points used for active and chronic infections may not be appropriate for developing countries with a high infectious disease burden [63], such as Kigali, Rwanda. Elevated CRP levels were lower than those reported in pregnant Kenyan population and AGP slightly higher, although the gestational age range for the group including women in later stages of pregnancy potentially accounting for the differences [64]. Another study with Ugandan women at similar timing of gestation had comparable elevated AGP levels but lower CRP ones [65].

\section{Maternal sociodemographics}

Maternal education level (primary schooling vs. no schooling) was inversely associated with maternal leukocyte telomere length. Previous studies involving pregnant women have reported that higher education level is associated with longer leukocyte telomere length and is a potential good proxy for socioeconomic status [66]. It remains unclear as to why primary education was associated with shorter leukocyte telomere length in our study population after adjusting for age and other risk factors. It is possible that unknown confounders are associated with higher maternal education levels that additionally adversely impact leukocyte telomere length in Rwandan women. Further studies are warranted to comprehend the relationship between education level and health outcomes in East African populations.

\section{Maternal obstetrical history}

We found no association among birth outcomes, including birth weight, low birth weight, and preterm birth, maternal reproductive history, and maternal leukocyte telomere length. Few studies have investigated these associations, but to our knowledge, none have been conducted in SSA. A study involving European mothers reported an association between shorter telomere length and intrauterine growth restriction [67], and previous studies have reported mixed results on the association between gravidity, parity, and maternal telomere length $[68,69]$.

\section{Conclusions}

We found associations between maternal age and micronutrients levels in early pregnancy and leukocyte telomere length in Rwandan women. Specifically, similar to other studies, we found that older maternal age was associated with shorter leukocyte telomere length even within a relatively narrow age range for women of reproductive age. We additionally found an association between markers of serum iron levels including higher ferritin levels and transferrin saturation receptor counts and shorter telomere length as well as lower retinol binding protein levels and shorter telomere length possibly suggestive of maternal health in pregnancy or alternatively specific to the environmental context of Rwanda. As the relationship between nutrition and infectious disease involves a feedback loop with infections affecting nutritional status and nutritional deficiencies impacting immune response and susceptibility to infections; further studies in this area are needed in developing countries.

\section{Limitations}

Ours is the first study to assess leukocyte telomere length in Rwanda. Additional studies are needed with more diverse population groups including men and children. Studies with pregnant women should more comprehensively explore maternal nutritional health and leukocyte telomere length in terms of nutritional 
assessments, including analyzing the intake of vitamin D, selenium, vitamin $C$, and other vitamins and minerals, in relation to maternal leukocyte telomere length outcomes. Future studies should also include multiple measurements of leukocyte telomere length during pregnancy, in addition to neonatal assessments and multiple nutritional and anthropometric maternal measurements throughout different periods of gestation.

Studies should also investigate the impact of prepregnancy BMI and maternal weight gain during pregnancy on maternal and neonatal outcomes including leukocyte telomere length as an outcome. Other studies should also comprehensively investigate infectious disease history including history of infection with malaria, STI history and other infectious disease burden including tuberculosis, hepatitis and dengue.

\section{Public health implications}

Nutritional intake during pregnancy is an actionable area for public health interventions in Rwandan women and children. Thus, further studies should assess the relationship between high iron levels, retinol binding protein and other markers of vitamin A status and shorter maternal leukocyte telomere length in the context of endemic infectious diseases. It is possible that the current regimens of iron and multivitamin supplementation during pregnancy do not confer the same benefits in all pregnant women, particularly those living in areas with a high incidence of malaria or a high burden of other infectious diseases.

\section{Abbreviations \\ AGP: a1-acid glycoprotein; BMI: Body mass index; Cl: Confidence interval; CRP: C-reactive protein; HIV: Human immunodeficiency virus; MUAC: Mid- upper arm circumference; RBP: Retinol-binding protein; ROS: Reactive oxygen species; SSA: Sub-Saharan Africa; sTfR: Soluble transferrin receptor; WHO: World Health Organization}

\section{Acknowledgements}

We thank the staff members at health facilities in Gasabo for their collaboration. We are also thankful to all participants and collaborators.

\section{Authors' contributions}

EN and JW conceptualized the overall study; EN, AU, CMM, and SR, JW was the promotor of leucocyte telomeres length substudy and designed the study protocol together with EN and NS, PM, and NM revised the protocol. $\mathrm{AU}$ and DN contributed to data collection. EN, JW, and NS contributed to data analysis. EN, JW JLC MN and EJC drafted and edited the manuscript; JL conducted telomere length analysis and edited the manuscript. All authors reviewed, edited, contributed, and approved the final version of the manuscript.

\section{Funding}

This study was funded by the East Africa Preterm Birth Initiative, a multiyear, multi-country effort generously funded by the Bill and Melinda Gates Foundation, and the funding covered publication charges (investment ID: OPP1107312). The funder had no role in study design; data collection, analysis or interpretation; or writing the manuscript.

Further, laboratory work involving leukocyte telomere length analysis was funded by the Nutrition and Obesity Research Center (NORC) at the University of California, San Francisco (P30DK098722).

\section{Availability of data and materials}

Available with the corresponding author (etiennen70@gmail.com) and will be deposited in a public repository as soon as we gain permission to do so.

\section{Ethics approval and consent to participate}

The study protocol was approved by the Rwanda Ministry of Health and the Institutional Review Board at the College of Medicine and Health Sciences (University of Rwanda) (approval notice: No213/CMHS IRB/2017) and the Committee on Human Research (Institutional Review Board) of the University of California, San Francisco. All participants provided signed, informed consent prior to participation in the study and were allowed to terminate participation at any time point during the study's duration.

\section{Consent for publication}

Not applicable.

\section{Competing interests}

The authors declare that they have no competing interests.

\section{Author details}

${ }^{1}$ College of Medicine and Health Sciences School of Health Sciences, University of Rwanda, P.O. Box: 3538, Kigali, Rwanda. ${ }^{2}$ College of Medicine and Health Sciences School of Medicine and Pharmacy, University of Rwanda, P.O. Box: 3538, Kigali, Rwanda. ${ }^{3}$ Emory University, Nell Hodgson Woodruff School of Nursing, Atlanta, Georgia, USA. ${ }^{4}$ Columbia University School of Nursing, New York, NY 10032, USA. ${ }^{5}$ University of California San Francisco, Institute for Global Health Sciences, San Francisco, USA. ${ }^{6}$ Department of Biochemistry and Biophysics, University of California San Francisco, San Francisco, USA. Departmentof Family Health Care Nursing, University of California San Francisco, San Francisco, USA. ${ }^{8}$ University of Rwanda College of Medicine and Health Sciences School of Public Health, P.O. Box: 3538, Kigali, Rwanda. ${ }^{9}$ Department of Pediatrics, University of California San Francisco, San Francisco, USA. ${ }^{10}$ Department of Epidemiology and Biostatistics, University of California, 550 16th Street, San Francisco, CA 941558, USA.

Received: 18 May 2020 Accepted: 9 October 2020

Published online: 13 November 2020

\section{References}

1. Lazarides C, Epel ES, Lin J, Blackburn EH, Voelkle MC, Buss C, et al. Maternal pro-inflammatory state during pregnancy and newborn leukocyte telomere length: a prospective investigation. Brain Behav Immun. 2019;80(April):41926. https://doi.org/10.1016/j.bbi.2019.04.021.

2. Opresko PL, Shay JW. Telomere-associated aging disorders. Ageing Res Rev. 2017;33:52-66. https://doi.org/10.1016/j.arr.2016.05.009.

3. Robertson T, Batty GD, Der G, Fenton C, Shiels PG, Benzeval M. Is socioeconomic status associated with biological aging as measured by telomere length? Epidemiol Rev. 2013;35(1):98-111. https://doi.org/10.1093/ epirev/mxs001.

4. Rizvi S, Raza ST, Mahdi F. Telomere length variations in aging and agerelated diseases. Curr Aging Sci. 2015;7(3):161-7.

5. Berti C, Biesalski HK, Gärtner R, Lapillonne A, Pietrzik K, Poston L, et al. Micronutrients in pregnancy: current knowledge and unresolved questions. Clin Nutr. 2011;30(6):689-701.

6. Pangrazzi L. Boosting the immune system with antioxidants: where are we now? Biochem (Lond). 2019;41:42-4.

7. Huang $Z$, Rose AH, Hoffmann PR. The role of selenium in inflammation and immunity: From molecular mechanisms to therapeutic opportunities. Antioxidants Redox Signal. 2012;16(7):705-43.

8. Marra MV, Drazba MA, Holásková I, Belden WJ. Nutrition risk is associated with leukocyte telomere length in middle-aged men and women with at least one risk factor for cardiovascular disease. Nutrients. 2019;11(3):1-16.

9. Entringer S, Epel ES, Blackburn EH, Buss CSB, et al. Maternal folate concentration in early pregnancy and newborn telomere length. Physiol Behav. 2017;176(1):139-48.

10. Myers KO, Ibrahimou B, Yusuf KK, Mauck DE, Salihu HM. The effect of maternal vitamin C intake on fetal telomere length. J Matern Neonatal Med. 2019;1-6. https://doi.org/10.1080/14767058.2019.1628940. 
11. Desyibelew HD, Dadi AF. Burden and determinants of malnutrition among pregnant women in Africa: a systematic review and meta-analysis. PLoS One. 2019;14(9):1-19.

12. Ryan Wessells K, Ouédraogo CT, Young RR, Thierno Faye M, Brito A, Hess SY. Micronutrient status among pregnant women in zinder, niger and risk factors associated with deficiency. Nutrients. 2017;9(5).

13. Hakizimana D, Nisingizwe MP, Logan J, Wong R. Identifying risk factors of anemia among women of reproductive age in Rwanda - a cross-sectional study using secondary data from the Rwanda demographic and health survey 2014/2015. BMC Public Health. 2019;19(1):1-11.

14. National Institute of Statistics of Rwanda (NISR) M of, Health (MOH) [Rwanda] II. Rwanda demographic and health survey 2014-15 final report. 2016. 307 p. Available from: https://dhsprogram.com/pubs/pdf/FR316/ FR316.pdf.

15. Nsereko E, Uwase A, Mukabutera A, Muvunyi CM, Rulisa S, Ntirushwa D, et al. Maternal genitourinary infections and poor nutritional status increase risk of preterm birth in Gasabo District, Rwanda: a prospective, longitudinal, cohort study. BMC Pregnancy Childbirth. 2020;20(1):345.

16. Joseph Davey DL, Nyemba DC, Gomba Y, Bekker LG, Taleghani S, DiTullio DJ, et al. Prevalence and correlates of sexually transmitted infections in pregnancy in HIV-infected and- uninfected women in Cape Town, South Africa. PLoS One. 2019;14(7):1-14

17. Alemu A, Moges F, Shiferaw Y, Tafess K, Kassu A, Anagaw B. Bacterial profile and drug susceptibility pattern of urinary tract infection in pregnant women at University of Go. BMC Res Notes. 2012;5(197):7. Available from: https:// www.ncbi.nlm.nih.gov/pmc/articles/PMC3473254/pdf/1756-0500-5-197.pdf

18. Mutagoma M, Balisanga H, Malamba SS, Sebuhoro D, Remera E, Riedel DJ, et al. Hepatitis B virus and HIV co-infection among pregnant women in Rwanda. BMC Infect Dis. 2017;17(1):1-7.

19. President's Malaria Initiative. Operational plan for Rwanda. FY 2017. 2017;1-72.

20. USAID, CDC, USA Department of State, USA Department of Health and Human Services. Rwanda - malaria operational plan FY 2018. 2018:54. Available from: https://reliefweb.int/sites/reliefweb.int/files/resources/fy-201 8-rwanda-malaria-operational-plan.pdf.

21. Sauder MW, Lee SE, Schulze KJ, Christian P, Wu LSF, Khatry SK, et al. Inflammation throughout pregnancy and fetal growth restriction in rural Nepal. Epidemiol Infect. 2019;147:e258.

22. Lee ACC, Quaiyum MA, Mullany LC, Mitra DK, Labrique A, Ahmed P, et al. Screening and treatment of maternal genitourinary tract infections in early pregnancy to prevent preterm birth in rural Sylhet, Bangladesh: a cluster randomized trial. BMC Pregnancy Childbirth. 2015;15(1):1-14.

23. Erhardt JG, Estes JE, Pfeiffer CM, Biesalski HK, Craft NE. Combined measurement of ferritin, soluble transferrin receptor, retinol binding protein, and C-reactive protein by an inexpensive, sensitive, and simple sandwich enzyme-linked immunosorbent assay technique. J Nutr. 2018;134(11):3127-32.

24. World Health Organization. Haemoglobin concentrations for the diagnosis of anaemia and assessment of severity. Miner Nutr Inf Syst World Health Organ. 2013;1-6.

25. Thurnham DI, Mccabe LD, Haldar S, Wieringa FT, Northrop-clewes CA, Mccabe GP. Adjusting plasma ferritin concentrations to remove the effects of subclinical inflammation in the assessment of iron deficiency: 2018; (August):546-55

26. Cawthon RM. Telomere measurement by quantitative PCR. Nucleic Acids Res. 2002;30(10):10-47.

27. Jue Lin, Epel ECJ, et al. Analyses and comparisons of telomerase activity and telomere length in human T and B cells: insights for epidemiology of telomere maintenance. J Immunol Methods. 2010;3521(1):71-80.

28. Rao DSR, Pindi DKG, Rani DU, Sasikala DG, Kawle DV. Diagnosis of bacterial vaginosis: Amsel's criteria vs Nugent's scoring. Sch J Appl Med Sci. 2016;4(6):2027-31.

29. Patil M, Nagamoti J, Metgud S. Diagnosis of Trichomonas vaginalis from vaginal specimens by wet mount microscopy, in pouch TV culture system, and PCR. J Glob Infect Dis. 2012:4(1):22.

30. Babić M, Hukić M. Candida albicans andon-albicans species as etiological agent of vaginitis in pregnant and non-pregnant women. Bosn J Basic Med Sci. 2010;10(1):89-97.

31. Hislop J, Quayyum Z, Flett G, Boachie C, Fraser C, et al. Systematic review of the clinical effectiveness and cost-effectiveness of rapid point-of-care tests for the detection of genital chlamydia infection in women and men. Health Technol Assess (Rockv). 2010;14(29):i-125. https://doi.org/10.3310/hta14290. Available from: http://www.embase.com/search/results?subaction= viewrecord\&from $=$ export\&id=L359134054.
32. Bartlett JG. Laboratory diagnosis of urinary tract infections in adult patients. Infect Dis Clin Pract. 2004;12(6):360-1.

33. Venter SN, Lotter HS, de H D. and M. The use of the analytical profile index in the identification of activated sludge bacteria: Problems and solutions. Water SA. 2000;15(4):4738.

34. FAO and FHI 360. Minimum dietary diversity for women- a guide to measurement. 2016. 82 p.

35. Martin-Prevel Y, Arimond M, Allemand P, Wiesmann D, Ballard TJ, Deitchler $\mathrm{M}$, et al. Development of a dichotomous indicator for population-level assessment of dietary diversity in women of reproductive age. Curr Dev Nutr. 2017:1(12):cdn.117.001701.

36. World Health Organization. Obesity: preventing and managing the global epidemic - WHO technical report series. WHO Technical Report Series. 2000

37. Tang AM, et al. Determining a global mid-upper arm circumference cutoff to assess malnutrition in pregnant women. Washington, DC; 2016

38. Saberi S, Kalloger SE, Zhu MMT, Sattha B, Maan EJ, van Schalkwyk J, et al. Dynamics of leukocyte telomere length in pregnant women living with HIV, and HIV-negative pregnant women: a longitudinal observational study. PLoS One. 2019;14(3):1-20.

39. Wong JYY, De Vivo I, Lin X, Fang SC, Christiani DC. The relationship between inflammatory biomarkers and telomere length in an occupational prospective cohort study. PLoS One. 2014;9(1).

40. Kateera F, Nsobya SL, Tukwasibwe S, Mens PF, Hakizimana E, Grobusch MP, et al. Malaria case clinical profiles and Plasmodium falciparum parasite genetic diversity: a cross sectional survey at two sites of different malaria transmission intensities in Rwanda. Malar J. 2016;15(1):1-10.

41. Aubert G, Lansdorp PM. Telomeres and aging. Physiol Rev. 2008;88(2):557-79.

42. Mather KA, Jorm AF, Parslow RA, Christensen $\mathrm{H}$. Is telomere length a biomarker of aging? A review. J Gerontol Ser A Biol Sci Med Sci. 2011;66(2):202-13. A(

43. Kuo CL, Pilling LC, Kuchel GA, Ferrucci L, Melzer D. Telomere length and aging-related outcomes in humans: a Mendelian randomization study in 261,000 older participants. Aging Cell. 2019;18(6):1-12.

44. Hansen MEB, Hunt SC, Stone RC, Horvath K, Herbig U, Ranciaro A, et al. Shorter telomere length in Europeans than in Africans due to polygenetic adaptation. Hum Mol Genet. 2016;25(11):2324-30.

45. Katherine A, Ahrens, Lauren M, Rossen AES. Relationship between mean leucocyte telomere length and measures of allostatic load in US reproductive-aged women, NHANES 1999-2002. Paediatr Perinat Epidemiol. 2016:30(4):325-35.

46. Liu B, Sun $Y, X u$ G, Snetselaar LG, Ludewig G, Wallace RB, et al. Association between body iron status and leukocyte telomere length, a biomarker of biological aging, in a nationally representative sample of US adults. J Acad Nutr Diet. 2019;119(4):617-25. https://doi.org/10.1016/j.jand.2018.09.007.

47. Shin C, Baik I. Transferrin saturation concentrations associated with telomeric ageing: a population-based study. Br J Nutr. 2017;117(12):1693-701.

48. Mazidi M, Kengne AP, Banach M. Mineral and vitamin consumption and telomere length among adults in the United States. Polish Arch Intern Med. 2017;127 127(2 2):87-90

49. Kabyemela ER, Fried M, Kurtis JD, Mutabingwa TK, Duffy PE. Decreased susceptibility to plasmodium falciparum infection in pregnant women with iron deficiency. J Infect Dis. 2008;198(2):163-6.

50. Kepinska M, Szyller J, Milnerowicz H. The influence of oxidative stress induced by iron on telomere length. Environ Toxicol Pharmacol. 2015:40(3): 931-5. https://doi.org/10.1016/j.etap.2015.10.002.

51. Ellervik C, Marott JL, Tybjærg-Hansen A, Schnohr P, Nordestgaard BG. Total and cause-specific mortality by moderately and markedly increased ferritin concentrations: general population study and metaanalysis. Clin Chem. 2014:60(11):1419-28.

52. Paul L. Diet, nutrition and telomere length. J Nutr Biochem. 2011;22(10): 895-901. https://doi.org/10.1016/j.jnutbio.2010.12.001.

53. Qun X, Parks CG, DeRoo LA, Cawthon RM, Sandler DP, Chen H. Multivitamin use and telomere length in women. Am J Clin Nutr. 2009:89(6):1857-63.

54. Maia SB, Souza ASR, Caminha MDFC, da Silva SL, Cruz R, de SBLC, Dos Santos CC, et al. Vitamin A and pregnancy: a narrative review. Nutrients. 2019;11(3):1-18.

55. Zeng Y, Li Y, Xia H, Wang S, Zhou J, Chen D. Retinoids, anxiety and peripartum depressive symptoms among Chinese women: a prospective cohort study. BMC Psychiatry. 2017;17(1):1-6.

56. Rode L, Nordestgaard BG, Weischer M, Bojesen SE. Increased body mass index, elevated C-reactive protein, and short telomere length. J Clin Endocrinol Metab. 2014;99(9):E1671-5. 
57. Gielen M, Hageman GJ, Antoniou EE, Nordfjall K, Mangino M, Balasubramanyam $\mathrm{M}$, et al. Body mass index is negatively associated with telomere length: a collaborative cross-sectional meta-analysis of 87 observational studies. Am J Clin Nutr. 2018;108(3):453-75.

58. Salvestrini V, Sell C, Lorenzini A. Obesity may accelerate the aging process. Front Endocrinol (Lausanne). 2019;10(MAY):1-16.

59. Pathai S, Lawn SD, Gillbert CE, McGuinness D, McGlynn L, Weiss HA, et al. Accelerated biological ageing in HIV-infected individuals in South Africa: a case-control study. Aids. 2013;27(15):2375-84.

60. Côte HCF, Hsieh AYY. Leukocyte telomere length in HIV infection: a marker of persistent immune aging or transient immune reconstitution? I Infect Dis. 2018;218(10):1521-2.

61. Aiello AE, Jayabalasingham B, Simanek AM, Diez-Roux A, Feinstein L, Meier HCS, et al. The impact of pathogen burden on leukocyte telomere length in the multi-ethnic study of atherosclerosis. Epidemiol Infect. 2017;145(14):3076-84.

62. Gannon BM, Glesby MJ, Finkelstein JL, Raj T, Erickson D, Mehta S. A point-ofcare assay for alpha-1-acid glycoprotein as a diagnostic tool for rapid, mobile-based determination of inflammation. Curr Res Biotechnol. 2019;1: 41-8. https://doi.org/10.1016/j.crbiot.2019.09.002.

63. Kohler IV, Anglewicz P, Kohler HP, McCabe JFCB. and BS. Evaluating health and disease in Sub-Saharan Africa: minimally invasive collection of plasma in the Malawi Longitudinal Study of Families and Health (MLSFH). Genus. 2012;68(2):1-27.

64. Mwangi MN, Echoka E, Knijff M, Kaduka L, Werema BG, Kinya FM, et al. Iron status of Kenyan pregnant women after adjusting for inflammation using brinda regression analysis and other correction methods. Nutrients. 2019; 11(2).

65. Baingana RK, Enyaru JK, Tjalsma H, Swinkels DW, Davidsson L. The aetiology of anaemia during pregnancy: a study to evaluate the contribution of iron deficiency and common infections in pregnant Ugandan women. Public Health Nutr. 2015;18(8):1423-35.

66. Mitchell AM, Kowalsky JM, Epel ESLJ. and LC. Child adversity, social support, and telomere length among perinatal women. Psychoneuroendocrinology. 2018;87:43-52

67. Perales-Puchalt A, Soberón N, Monterde M, Hervas-Marin D, Foronda M, Desantes $\mathrm{D}$, et al. Maternal telomere length is shorter in intrauterine growth restriction versus uncomplicated pregnancies, but not in the offspring or in IVF-conceived newborns. Reprod Biomed Online. 2019;38(4):606-12.

68. Lane-Cordova AD, Puterman E, Gunderson EP, Chan C, Hou L, Carnethon M. Gravidity is not associated with telomere length in a biracial cohort of middle-aged women: The Coronary Artery Risk Development in Young Adults (CARDIA) study. PLoS One. 2017:12(10):1-11.

69. Pollack AZ, Rivers K, Ahrens KA. Parity associated with telomere length among US reproductive age women. Hum Reprod. 2018;33(4):736-44.

\section{Publisher's Note}

Springer Nature remains neutral with regard to jurisdictional claims in published maps and institutional affiliations.

Ready to submit your research? Choose BMC and benefit from:

- fast, convenient online submission

- thorough peer review by experienced researchers in your field

- rapid publication on acceptance

- support for research data, including large and complex data types

- gold Open Access which fosters wider collaboration and increased citations

- maximum visibility for your research: over $100 \mathrm{M}$ website views per year

At $\mathrm{BMC}$, research is always in progress.

Learn more biomedcentral.com/submissions 